\title{
Effectiveness, safety and cost analysis of add-on pidotimod in maintenance therapy for exacerbation of chronic obstructive pulmonary disease in adults
}

\author{
Ashish Goyal \\ Consultant, Dept. of Pulmonary Medicine, Government Medical College and Hospital, Nagpur, Maharashtra, India
}

*Corresponding Author:

Email: vejayaaol@gmail.com

\begin{abstract}
Acute exacerbation of chronic obstructive pulmonary disease (AECOPD) is the sudden worsening in airway function and respiratory symptoms in patients with COPD which range from self-limited diseases to episodes of florid respiratory failure requiring mechanical ventilation. An average patient with COPD might experience 2 episodes of AECOPD per year and $10 \%$ of these episodes require hospitalization. Pidotimod, an immunostimulant, has shown promising results in conditions with underlying cause of suppressed cell-mediated immunity like chronic bronchitis and recurrent respiratory tract infection (RRTI). The current study evaluated the safety, efficacy and cost effectiveness of Pidotimod in adult Indian patients with COPD as an add-on drug in maintenance therapy of acute exacerbations. In this prospective, open label, single arm, single centre study, Pidotimod was administered as an add-on therapy for 2 months in 114 patients ( $\geq 18$ years and $\leq 55$ years; mean age: $59.51 \pm 8.66$ years) with COPD having experienced two or more exacerbation that required antibiotics. The study comprised of screening visit, enrolment and 3 follow-up visits (at the end of 2, 6 and 12 months). Of the 111 patients at baseline, only one patient had an AECB episode at 2 and 6 months. At the end of 12 months, none of the patient had an AECB. Only one subject was prescribed with antibiotic treatment at the end of 2 and 6 months. No episodes of either exacerbations or antibiotic prescription at the end of 12 months were reported. A significant reduction $(\mathrm{p}<0.05)$ in mean modified British Medical Research Council $(\mathrm{mMRC})$ score was observed at the end of 6 and 12 months from baseline. There were no hospitalizations at any of the follow-up visits. Only one patient (0.9\%) was taking reliever/rescue medication. The mean expenses significantly reduced at the end of 2 and 6 months of treatment with Pidotimod. Pidotimod was well tolerated. In conclusion, Pidotimod is safe, effective and cost effective as maintenance therapy for exacerbation of COPD, when added to the standard of care.
\end{abstract}

Keywords: Immunostimulant, Pidotimod, Acute exacerbations of chronic bronchitis (AECB), Cost-analysis, Respiratory infections.

\section{Introduction}

Chronic obstructive pulmonary disease (COPD) is a progressive and non-reversible respiratory illness characterized by airflow limitation and exacerbations. It is a major cause of morbidity and mortality worldwide[1,2]. The exacerbations are mostly caused by either viral upper respiratory tract infections (URTI) in COPD or due to bacteria. S. pneumoniae, H. influenzae, $H$. parainfluenzae, $M$. catarrhalis and $P$. aeruginosa and resistant Gram-negative bacteria are the dominant pathogens in severe exacerbations[3]. Additionally, host factors, i.e., genetic abnormalities, abnormal lung development and accelerated aging predispose an individual to COPD [4]. COPD affects around 210 million people worldwide and is predicted to be the third leading cause of death worldwide by 2020[1,5]. According to the World Health Organization (WHO) fact sheet (2017), and the Global Burden of Disease Study report, a prevalence of 251 million cases of COPD was reported globally in 2016[6]. The low and middle income countries contribute to $>90 \%$ of COPD deaths with only India and China contributing to $66 \%$ deaths[3]. As per the Global Burden of Diseases, Injuries, and Risk Factors Study (GBD) 2016done from 1990 to 2016, the contribution of chronic respiratory diseases to the total deaths and disability-adjusted life-years (DALYs ) in
India increased from $4 \cdot 5 \%(95 \%$ UI $4 \cdot 0-4 \cdot 9)$ in 1990 to $6 \cdot 4 \%(5 \cdot 8-7 \cdot 0)$ in 2016[7].

There is an evidence which indicates that components of innate immunity in COPD, i.e. those provided by the airway epithelial barrier, including alveolar macrophages, neutrophils, dendritic cells and natural killer cells, are broadly suppressed in COPD[8]. Adaptive immune responses elicited by the lung tissue antigens in the lungs of patients with COPD, with the participation of cytotoxic $\mathrm{CD} 8+\mathrm{T}$ cells, T helper 1, Th17 CD4+ cells, and B-cell responses with antibody production aggravates the condition. Percentage of CD8+ T cells expressing toll like Receptors (TLRs) is significantly increased in patients with COPD. Also, TLR2/1 and TLR2 on lung CD4+ T cells and CD8+ NKT cells, respectively, show a significant increase in COPD $[9,10]$.

Antimicrobial therapy, bronchodilators such as long-acting muscarinic antagonist (LAMA), long-acting beta agonist (LABA), inhaled corticosteroids (ICS)are the conventionally prescribed treatment in the management of COPD[11, 12]. Despite the availability of diverse treatment options, the disease is still widely affecting adults. This could be due to the antibiotic resistance or due to immune-deficiencies in the individuals $[13,14]$. There are still many unmet needs in the management of COPD for both patients and health 
providers. Hospitalization needs due to frequent and potentially life threatening exacerbations, and expensive treatment add financial burden to the patient and family for disease management and treatment of exacerbations[12]. COPD exacerbations alone account for the greatest proportion of total COPD burden on the healthcare system. Therefore, there is a need to develop cost-effective treatment strategy for patients with severe COPD who continue to exacerbate despite existing bronchodilator and antimicrobial therapies.

Studies from various clinical trials and expert opinion reports have shown that the immunosuppressants (IS) are effective in management of COPD in comparison to other conventional treatment $[15,16]$. IS such as cyclosporine, tacrolimus, CpG (TLR9 agonists) and few monoclonal antibodies have shown effective results in treating AECB by targeting the prime inflammatory pathways[17,18]. In adults, IS are the suitable choice as monotherapy or in combination with antibiotic therapy[19,20].

Pidotimod (3-L-pyroglutamyl-L-thiaziolidine-4 carboxylic acid) is a synthetic dipeptide molecule with immune modulatory properties[21]. It eliminates infection symptoms faster, promotes the recovery and allows the reduction of concomitant medications[22]. Its potential for immune stimulation has been evaluated in conditions with underlying cause of suppressed cellmediated immunity to certain extent, such as chronic bronchitis and recurrent respiratory tract infection (RRTI)in children and adults[13,21,23,24].

Previous studies suggested that Pidotimod is clinically effective in the treatment of exacerbations and helps in better and faster remission of symptoms by repairing and improving immune responses[25,26]. However, there is a lack of data from India. The current study evaluated the safety, efficacy and cost effectiveness of Pidotimod in adult Indian patients with COPD as an add-on drug in maintenance therapy of acute exacerbations.

\section{Materials and Methods Study Design and Setting}

This was a prospective, open label, single arm, single centre study conducted at Life point Research LLP /Life point Multispecialty Hospital, Pune, Maharashtra, India from 27 June 2016 to 03 February 2018. The study was performed in accordance with approved protocol, applicable local regulations and international guidelines. All the study participants were provided a freely obtained written informed consent before participation in the study. The study was compliant to the good clinical practices $(\mathrm{GCP})$ guidelines for clinical trial on pharmaceutical products in India and ethics of biomedical research, ministry of health and family welfare. The study was conducted as per Declaration of Helsinki for biomedical research on human subjects[27].

\section{Study Subjects}

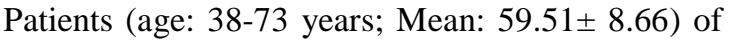
either gender diagnosed with COPD according to the GOLD criteria having experienced two or more exacerbation of COPD that required antibiotics for therapy or one or more exacerbation requiring hospitalization in last 12 months were included. The included patients were required to be clinically stable (no episode of exacerbation) during one month prior to enrolment into study and have FEV $1 \leq 80 \%$ and $\geq 30 \%$. Patients with hypersensitivity/allergy to the study medication, those on steroid therapy, i.e., $>10 \mathrm{mg}$ of prednisone a day for more than 1 week within a month prior to enrolment in the study; patients with asthma (defined as episodic symptoms of airflow obstruction which was reversible with bronchodilators, assessed with lung function testing) were excluded from the study.

\section{Study Visits/Schedule}

This was a 21 months study conducted from June 2016 to February 2018 (6 months for enrolment, 12 months of study participation and 3 months of data management and analysis) comprised of five visits. Following a screening visit (Visit 1, Day -7 to Day 0), the patients were enrolled (Visit 2, Day 1). The patients were followed at the end of 2 (Visit 3), 6 (Visit 4) and 12 (Visit 5) months (Fig. 1). During the screening, patient's demographics, medical history, smoking history, concomitant medications, complete physical examination, and baseline laboratory assessment was conducted. At every follow-up visit, complete details of any exacerbations occurring post enrolment, medical expenses incurred (direct and indirect), smoking history, and concomitant medications were recorded. A complete physical examination, administration of modified British Medical Research Council (mMRC),St. George's Respiratory Questionnaire (SGRQ)[28] and Pulmonary function testing (FVC, FEV1 and any other) along with complete haematology and biochemistry were conducted during all these follow-up visits.

In addition, patients were followed up on telephone at the end of 4 and 9 months for details of any AECB since the last visit. All the subjects were advised to report any adverse events (AEs) encountered during the study period. 


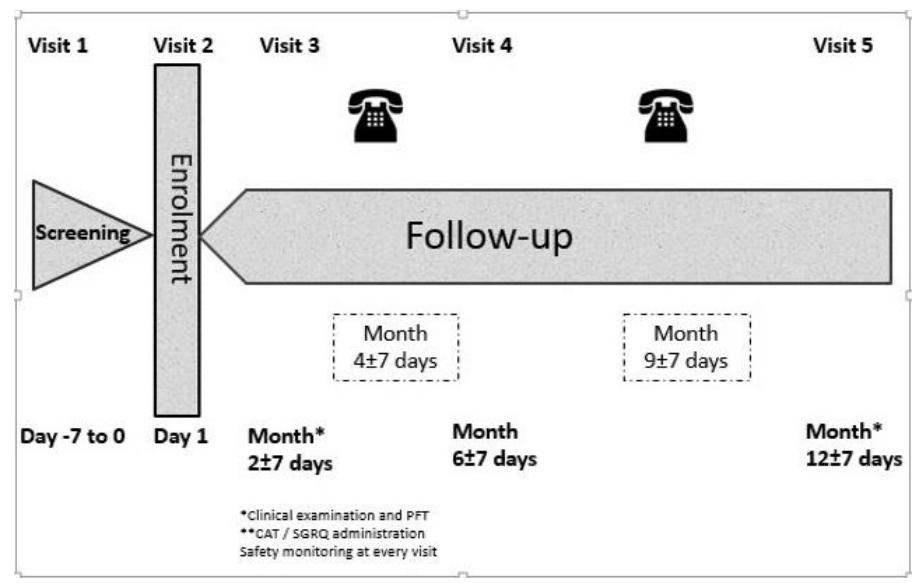

Fig. 1: Study Design

\section{Dosage Schedule}

Pidotimod was administered as an add-on therapy at a dose of $800 \mathrm{mg}$ twice daily for 8 days and continued at $800 \mathrm{mg}$ once daily till completion of 2 months of treatment to the enrolled patients.

\section{Safety and Efficacy Variables}

Reduction in the number of exacerbations of COPD in 12 months post commencement of Pidotimod treatment was the primary efficacy variable. Other evaluations included; average duration of each episode of exacerbation, number of episode requiring antibiotic therapy, average duration of antibiotic therapy required per episode, number of episodes requiring hospitalization, number of work days lost due to exacerbations of COPD, change in COPD symptom score as evaluated on modified British Medical Research Council (mMRC) and change in quality of life evaluated on SGRQ. Evaluation of AEs, serious adverse events (SAEs) and concomitant medication evaluation were safety end points for the study. Change in cost of medical management (direct and indirect) and hospital expenses after therapy with Pidotimod was the end-point for costanalysis variable.

\section{Sample Size and Statistical Analysis}

For the study to have a power of the study of $95 \%$; a sample size of 108 was optimal. Assuming a drop-out rate of $10 \%$, total 119 patients were to be enrolled into the study. Safety analysis was done for safety population (patients who completed the entire study). For efficacy analysis, intention to treat (ITT; those who were enrolled in the study and received at least one dose of study drug). Last observation carried forward (LOCF) approach was used for missing values. Demographic data such as age, gender, weight, and other variables were summarized using descriptive statistics. Quantitative variables were expressed as mean, SD with range. Qualitative variables were presented as counts and percentage. Chi square test and Student t-test were used to analyse the data. Statistical package for social science (SPSS) (Version 10) software was used for statistical analysis.

\section{Results}

A total of 114 subjects (77 men; $67.5 \%$ and 37 women; $32.5 \%$ ) with a mean age of $59.51 \pm 8.66$ years (range: 38 to 73 years) received Pidotimod. Of these, 30 patients had duration of smoking ranging from 1 to 50 years, with an average of $20.57 \pm 11.88$ years. Smoking history of the patients ranged from $1-40$ packs with an average of $7.61 \pm 8.65$ packs. A total of $110(96 \%)$ of patients were on bronchodilator followed by $68(59.6 \%)$ on antibiotics.

Table 1 shows the demographic characteristics of patients recruited. Overall, 111 patients completed the study and were analysed for efficacy parameters. Three subjects were withdrawn as they were lost to follow up.

\section{Table 1: Demographic Characteristics of Patients}

\begin{tabular}{|l|l|}
\hline Age (years) & $59.51 \pm 08.66$ \\
Mean \pm SD & $38.00-73.00$ \\
\hline Range & $77(67.5)$ \\
\hline Sex $(\%)$ & $37(32.5)$ \\
Male & \\
Female & \\
\hline Smoking History $($ No. of packs) & \\
$\mathbf{( N = 3 1 )}$ & $07.61 \pm 08.65$ \\
Mean \pm SD & $01.00-40.00$ \\
Range & \\
\hline & \\
Years of smoking $(\mathbf{N}=\mathbf{3 0})$ & $20.57 \pm 11.88$ \\
Mean \pm SD & $01.00-50.00$ \\
Range & \\
\hline
\end{tabular}

\section{Efficacy Analysis (Fig. 2)}

1. Number of AECB Episodes and Prescription of Antibiotics after Treatment: The mean number of AECB episodes at baseline were $2.10 \pm 0.50$. Of the 111 patients at baseline, only one patient had an AECB episode at 2 and 6 months. None of the patient had an AECB at the end of 12 months.

The mean number of episodes of antibiotics prescribed at baseline were $2.08 \pm 0.56$. After treatment with Pidotimod, only one subject was 
prescribed with antibiotic treatment for 5 days each. The baseline duration of antibiotic treatment was $5.58 \pm 1.21$ days. There were no episodes of either exacerbations or antibiotic prescription at the end of 12 months.

2. Modified British Medical Research Council (mMRC) and St. George's Respiratory Questionnaire (SGRQ) Score: The mean mMRC score at baseline was $2.81 \pm 0.60$, which reduced to $2.65 \pm 0.97$ at the end of 2 months and significantly $(\mathrm{p}<0.05)$ reduced to $2.46 \pm 0.73$ and $2.47 \pm 0.67$ at the end of 6 and 12 months, respectively. There was an overall decrease in mMRC score after treatment with Pidotimod. The SGRQ score was 39.09 \pm 13.77 throughout the study $(\mathrm{p}=1.000)$.

i. FEV1 / FVC Ratio and Hospitalizations: There was no significant change $(\mathrm{p}>0.05)$ in FEV1 / FVC ratio from baseline to 2 months of treatment duration. At baseline, 4 patients $(4 / 114 ; 3.5 \%)$ were hospitalized and the average duration of hospitalization was $4.00 \pm$ 2.45. There were no hospitalizations at any of the follow-up visits during the study. Only one patient $(0.9 \%)$ was taking reliever / rescue medication.
Apart from the above efficacy parameters, we also observed changes in body weight from baseline as one of the secondary end points. At baseline the mean body weight was $63.85 \pm$ $12.51 \mathrm{Kg}$. After the treatment, the mean difference reported were $00.49 \pm 02.37 \mathrm{Kg}$, $00.48 \pm 01.98 \mathrm{Kg}$ and $00.46 \pm 01.92 \mathrm{Kg}$ at 2,6 and 12 months respectively. Mean difference was statistically significant at $6(\mathrm{p}=0.029)$ and $12(\mathrm{p}=0.031)$ months.

ii. Cost Analysis: Expenses after Treatment (Fig. 2 (b)) The mean expenses at baseline were Rs.1676.13, however, the data of only 28 patients was available after 2 months, which showed expenses of treatment significantly reduced to Rs. 919.64. After 6 months, the available data of only 5 patients showed mean expenses of Rs. 970.00 per patient.

* Significant $(\mathrm{p}<0.05)$

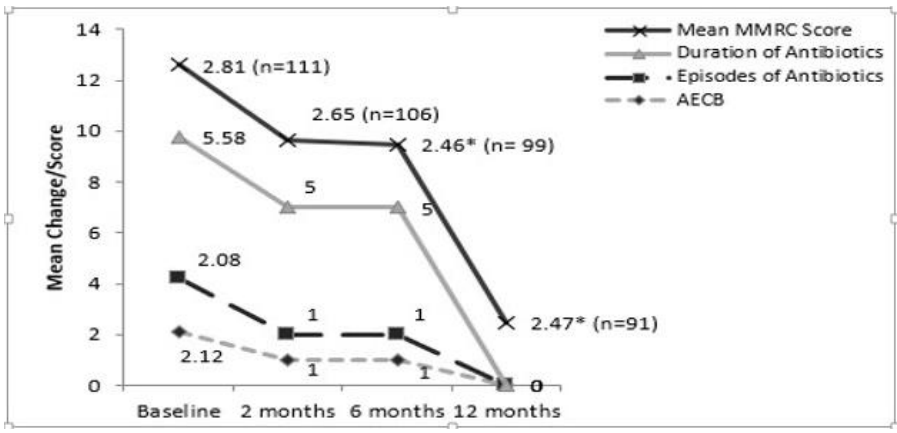

Fig. 2 (a): Efficacy results at the end of study: Number of AECB episodes and prescription of antibiotics after treatment and MMRC score

*Significant $(\mathrm{p}<0.05)$

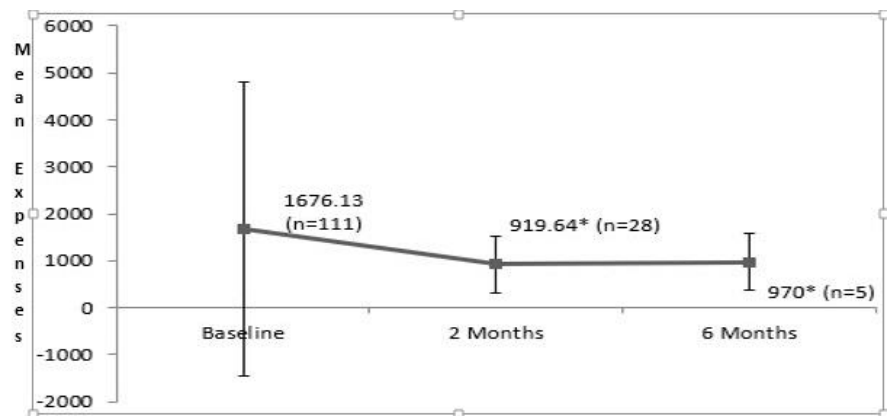

Fig. 2 (b): Efficacy Results: Cost Analysis

\section{Safety Analysis}

Amongst the 114 study patients, two patients reported SAEs and one with AE were observed. The AEs observed were pain in knees, body pain and weakness (Table 2). The intensity of events was moderate for all the cases, which was resolved during the treatment. None of the patients had to stop treatment because of AEs. No significant change 
in laboratory parameters such as LFT (SGPT and SGOT), total proteins, serum albumin, BUN, RFT (serum creatinine) and urine routine was observed among the study population. Two deaths (one due to acute on chronic respiratory failure and other of bilateral lung consolidation) were reported during the study which were not related to the study drug.

Table 2: Profile of Adverse Events (AEs)/ Serious Adverse Events (SAE) among Study Cases

\begin{tabular}{|c|c|c|}
\hline Adverse Events & $\begin{array}{c}\text { No. of Cases } \\
(\mathbf{N}=114)\end{array}$ & Percentage \\
\hline Patients with SAE & 02 & 01.8 \\
\hline Patients with at least one AEs & 01 & 00.9 \\
\hline \multicolumn{3}{|l|}{ Profile of Events $(n=3)$} \\
\hline Pain in knees & 01 & 00.9 \\
\hline Body pain & 01 & 00.9 \\
\hline Weakness & 01 & 00.9 \\
\hline
\end{tabular}

\section{Discussion}

Pidotimod induces dendritic cells (DCs) maturation, up-regulates the expression of HLADR and of costimulatory molecules, stimulates DCs to release proinflammatory molecules such as IL-2, TLRs and release of interferon (INF)- $\gamma[29,30]$. Pidotimod can be safely prescribed in patients with COPD even at a high risk of exacerbation[20].

The effectiveness of Pidotimod is demonstrated in adult patients $(\mathrm{n}=137)$ with chronic bronchitis of stage I, II and III. Pozzi et al. evaluated the effect of Pidotimod ( $800 \mathrm{mg}$ twice a day) in combination with antibiotics (amoxicillin and clavulanic acid $1 \mathrm{~g}$ twice daily) in comparison to placebo $(n=69)$ in adults and antibiotic group $(n=68)$ for a period of 45 days. Within 8 days of treatment, there was a decrease in sputum volume by $36.8 \%$ in the Pidotimod group. Changes of potentially pathogenic bacteria in sputum reduced to $8.1 \%$ in Pidotimod group and 9.7\% in placebo group[19]. Similarly, in another study of 16 adult patients with primary or metastatic neoplasm, Pidotimod proved to be clinically effective. A $12 \%$ increase in CD3 lymphocytes compartment was observed with a dose of 400 $\mathrm{mg} /$ day[24,26].

In India, there are limited clinical trials with Pidotimod (CTRI/2018/02/011898 and one on-going for Pidotimod in paediatric patients with ARTI and asthma)[31]. On an average, patients with COPD experience from one to four episodes of acute exacerbations (or acute bronchitis) per year. These episodes are the most common cause of death in COPD, and profoundly reduce the quality of life (QOL) of the patients. Despite the use of antibiotics, antipyretic drugs, and symptomatic drugs, the frequency of AECOPD is still high. A functional approach for AECOPD could thus be non-specifically increasing the immune response or enhancing the innate defence mechanisms; thus making the immunostimulant drugs an important strategic treatment approach to prevent and to attenuate infections.

Our study results showed that there was a decrease in the episodes of exacerbations from 2.12 at baseline to 1 at 6 months of treatment. A significant reduction was also observed in duration of episode, i.e., 5.54 days at baseline as compared to almost nil at the end of treatment. These results were consistent with similar studies based on immunomodulator based treatment in COPD. Study by Bruno et al., showed reduction in pathogen activity and recurrent exacerbations in COPD patients with immunomodulation effect of the treatment[32]. Similarly, Koatz et al, also found significantly lower rate of respiratory tract infections (74\% reduction) and exacerbations in COPD and asthma patients (36\% reduction) effect of drug ( OM-85 oral bacterial lysate) on immune system $(\mathrm{p}<0.05)$ [33].

In our study, $60 \%$ of patients were taking antibiotics during an exacerbation of COPD. We evaluated the change in the number of episodes of antibiotics prescribed and duration of antibiotics along with Pidotimod maintenance treatment. We reported the reduction in both the number of episodes and duration of antibiotic treatment. After 2 months of treatment, for only one patient antibiotic was prescribed for one episode and that was continued for follow up period of 6 months. None of the patient required antibiotic treatment after follow up of 12 months. These results are also in consistency with the results reported in one of the systematic reviews of controlled trials which concluded that treatment with immunomodulator was effective with regard to the average length of the exacerbations and antibiotic treatment used for the exacerbations. Both the variables declined significantly in the group treated with immunomodulator (AM3) (3.10 days and 8.07 days, $\mathrm{p}<$ 0.001 , respectively)[34]. This decline in use of concomitant medications also suggests that Pidotimod maintenance treatment is nevertheless a cost-effective treatment which could reduce the financial burden of expensive drugs.

We also evaluated other parameters such as change in COPD symptom score using mMRC and SGRQ questionnaire. SGRQ is the disease-specific instrument which was designed to measure impact on overall health, daily life, and perceived well-being in patients with obstructive airways disease. Various studies have used this questionnaire to measure the improvement in patients with respiratory disorders[35,36]. As per the 
mMRC and SGRQ scores, we reported a significant decrease in symptoms e.g. dyspnea, improvement in quality of life and decreased daily cost of treatment with Pidotimod. The findings suggest that the immune modulating effect of Pidotimod could be attributed to the positive results of mMRC and SGRQ.

Since acute exacerbations are the main cause of hospitalization among patients with COPD and approaches to prevent exacerbations could be very cost effective and may improve the QoL. In literature, there are few studies available to analyse the cost effectiveness of drugs used in COPD treatment. Studies done by Hertel et al., using Markov cohort model to predict lifetime costs, outcomes, and cost-effectiveness of various combinations of a long-acting muscarinic antagonist (LAMA), a long-acting beta agonist (LABA), an inhaled corticosteroid (ICS), and Roflumilast in a fully incremental analysis. Based on the results a costeffectiveness frontier was determined, indicating those treatment regimens which represent the most costeffectiveness approach[12]. The reduction in cost of treatment with Pidotimod reported in our study is in consistency with the conclusion of studies by Kallaru $e t$ $a l$., which states that there could be controlled cost of treatment if there is a reduction in the episodes of exacerbations[37]. Our results with Pidotimod cost effectiveness also showed a great fall in treatment expenses using Pidotimod as add on therapy; almost 50\% reduction from baseline to end of the treatment. However, the number of patients was less to derive any conclusive statement in this regard.

In the previous studies, Pidotimod has reported a good safety and tolerability profile[5]. Our study also confirmed a good safety profile for Pidotimod. Only 3 AEs were reported in the study; one patient reported $(0.9 \%)$ pain in knee, body pain and weakness and $1.8 \%$ were the SAEs which were unrelated to the drug. No new AEs were reported during the study. The deaths reported in the study were not related to Pidotimod treatment. Almost all the patients (97.3\%) completed the treatment without safety concerns.

\section{Conclusion}

A reduction in the overall number of exacerbations, reduced use of antibiotics, a significant reduction in duration of exacerbations episode from baseline to the end of treatment, reduced time to recovery and reduced hospitalizations strongly suggest use of Pidotimod as a promising treatment option for adult patients with AECOPD. To conclude, Pidotimod is a safe, efficacious, and cost-effective maintenance therapy for patients with AECOPD, when added to the standard of care.

\section{Conflict of interest: There is no conflict of interest}

\section{Acknowledgement}

The authors acknowledge Knowledge Isotopes Pvt. Ltd. (http://www.knowledgeisotopes.com) for the medical writing support. We are also thankful to Medical Affairs team of Wockhardt Ltd., Mumbai for their scientific support during planning and conduct of this research work as well as developing this manuscript.

\section{References}

1. Vestbo J, Hurd SS, Agustí AG, Jones PW, Vogelmeier C, et al. "Global Strategy for the Diagnosis, Management, and Prevention of Chronic Obstructive Pulmonary Disease". Am J Respir Critical Care Med 2013;187: 347 65.

2. Wedzicha JA, Seemungal TA, MacCallum PK, Paul EA, Donaldson GC, et al. "Acute exacerbations of chronic obstructive pulmonary disease are accompanied by elevations of plasma fibrinogen and serum IL-6 levels" Thrombosis haemostasis 2000;83:210-15.

3. Salvi SS, Manap R, Beasley R. "Understanding the true burden of COPD: the epidemiological challenges" Primary Care Respir J 2012;21:249.

4. "Global strategy for the diagnosis, management and prevention of chronic obstructive pulmonary disease (COPD). Available at URL: https://goldcopd.org/wpcontent/uploads/2017/11/GOLD-2018-v6.0-FINALrevised-20-Nov_WMS.pdf. Last accessed on 13 August 2018" (2018)

5. Zuccotti GV, Mameli C. "Respiratory infections and immunostimulants in childhood: an update". J Pediatr Neonatal Individualized Med 2015;4:e040218.

6. Rajkumar P, Pattabi K, Vadivoo S, Bhome A, Brashier B, et al. "A cross-sectional study on prevalence of chronic obstructive pulmonary disease (COPD) in India: rationale and methods" BMJ Open 2017;7.

7. Salvi S, Kumar GA, Dhaliwal R, Paulson K, Agrawal A, et al. "The burden of chronic respiratory diseases and their heterogeneity across the states of India: the Global Burden of Disease Study 1990-2016" The Lancet Global Health. (2018)

8. Shaykhiev R, Crystal RG. "Innate Immunity and Chronic Obstructive Pulmonary Disease - A Mini-Review" Gerontol (2013;59.

9. Bhat TA, Panzica L, Kalathil SG, Thanavala Y. "Immune Dysfunction in Patients with Chronic Obstructive Pulmonary Disease" Ann Am Thoracic Soc 2015;12:S169-S75.

10. Edwards MR, Saglani S, Schwarze J, Skevaki C, Smith JA, et al. "Addressing unmet needs in understanding asthma mechanisms: From the European Asthma Research and Innovation Partnership (EARIP) Work Package (WP) 2 collaborators" Eur Respir J 2017;49:1602448.

11. Hirschmann J. "Antibiotics for common respiratory tract infections in adults". Arch Internal Med 2002;162: 25664.

12. Hertel N, Kotchie RW, Samyshkin Y, Radford M, Humphreys S, et al. "Cost-effectiveness of available treatment options for patients suffering from severe COPD in the UK: a fully incremental analysis". Int $J$ Chronic Obstructive Pulmonary Dis 2012;7:183.

13. Ferrario BE, Garuti S, Braido F, Canonica GW. "Pidotimod: the state of art". Clin Molecular Allergy 2015;13:8

14. McNulty CA, Nichols T, French DP, Joshi P, Butler CC. "Expectations for consultations and antibiotics for respiratory tract infection in primary care: the RTI clinical iceberg" Br J Gen Pract. (2013) 63: e429-e36.

15. Collet J, Shapiro S, Ernst P, Renzi P, Ducruet T, et al. "Effects of an immunostimulating agent on acute 
exacerbations and hospitalizations in patients with chronic obstructive pulmonary disease" American journal of respiratory and critical care medicine. (1997) 156: 1719-24.

16. Minov J, Bislimovska-Karadzhinska J, Petrova T, Vasilevska K, Stoleski S, et al. "Effects of Pleuran (BGlucan from Pleurotus Ostreatus) Supplementation on Incidence and Duration of COPD Exacerbations" Open access Macedonian J Med Sci 2017;5:893.

17. Kim AS, Doherty TA. "New and emerging therapies for asthma" Ann Allergy Asthma Immunol 2016;116:14.

18. Ortega HG, Liu MC, Pavord ID, Brusselle GG, FitzGerald JM, et al. "Mepolizumab treatment in patients with severe eosinophilic asthma" New Engl J Med 2014;371:1198-207.

19. Pozzi E, Dolcetti A, Orlandi O, Cirianni C, Moreo G, et al. "Pidotimod in the treatment of patients affected by bacterial exacerbations of chronic bronchitis" Arzneimittel-Forschung. 1994;44:1495-8.

20. Del-Rio-Navarro B, Gonzalez-Diaz S, Jose Escalante Dominguez A, Blandon Vijil V. Immunostimulants in the prevention of respiratory infections 2007.

21. Zuccotti GV, Mameli C. "Pidotimod: the past and the present" Italian J Pediatr 2013;39:75.

22. Riboldi P, Gerosa M, Meroni P. Pidotimod: a reappraisal. SAGE Publications Sage UK: London, England; 2009.

23. Licari A, De MA, Nigrisoli S, Marseglia A, Caimmi S, et al. "Pidotimod may prevent recurrent respiratory infections in children" Minerva Pediatrica 2014;66:36367.

24. Benetti G, Illeni M, Passera A, Bombelli G, Lavecchia G, et al. "Ex vivo evaluation of pidotimod activity in patients with chronic obstructive pulmonary disease" Arzneimittel-forschung. 1994;44:1503-5.

25. Benetti G, Fugazza L, Stramba MB, Montalto F, Bombelli G, et al. "Ex vivo evaluation of pidotimod activity on cell-mediated immunity" Arzneimittelforschung 1994;44:1476-9.

26. Ciaccia A. "Pidotimod activity against chronic bronchitis exacerbations" Arzneimittel-Forschung 1994;44:1516-20.

27. PP R. "Human experimentation. Code of ethics of the world medical association. Declaration of Helsinki" $\mathrm{Br}$ Med J 1964;2:177.

28. Jones PW, Quirk F, Baveystock C. "The St George's respiratory questionnaire" Respir Med 1991;85:25-31.

29. Zuccotti G, Mameli C, Trabattoni D, Beretta S, Biasin M, et al. "Immunomodulating activity of Pidotimod in children with Down syndrome" J Biological Regulators Homeostatic Agents 2013;27:253-8.
30. Giagulli C, Noerder M, Avolio M, Becker PD, Fiorentini $\mathrm{S}$, et al. "Pidotimod promotes functional maturation of dendritic cells and displays adjuvant properties at the nasal mucosa level" Int Immunopharmacol 2009;9:136673.

31. Clinical trial: Effects of Pidotimod 400mg for repeated attacks of Respiratory Tract Infections in children. Available on URL:

http://www.ctri.nic.in/Clinicaltrials/pdf_generate.php?tria lid $=14356 \&$ EncHid $=\&$ modid $=\&$ compid $=\% 27, \% 2714356$ $\operatorname{det} \% 27$. Last accessed on 14 August 2018. [Internet]. 2018.

32. Bruno A, Cipollina C, Di Vincenzo S, Siena L, Dino P, et al. "Ceftaroline modulates the innate immune and host defense responses of immunocompetent cells exposed to cigarette smoke" Toxicol letters 2017;279:9-15.

33. Koatz AM, Coe NA, Ciceran A, Alter AJ. "Clinical and Immunological Benefits of OM-85 Bacterial Lysate in Patients with Allergic Rhinitis, Asthma, and COPD and Recurrent Respiratory Infections" Lung 2016;194:687-97.

34. Reyes Martin E, Fernandez Almendros C, Alvarez-Sala JL, Alvarez-Mon M. "[Effect of immunomodulator AM3 on the exacerbations in patients with chronic bronchitis: a systematic review of controlled trials]" Revista Clinica Espanola 2004;204:466-71.

35. Dalvi PS, Singh A, Trivedi HR, Ghanchi FD, Parmar DM, et al. "Effect of doxycycline in patients of moderate to severe chronic obstructive pulmonary disease with stable symptoms" Ann Thoracic Med 2011;6:221-26.

36. Wedzicha JA, Banerji D, Chapman KR, Vestbo J, Roche $\mathrm{N}$, et al. "Indacaterol-Glycopyrronium versus Salmeterol-Fluticasone for COPD" New Engl J Med 2016;374:2222-34.

37. Kallaru H, Nagasubramanian VR, Balakrishnan HP, Gopal K, Palani T. "Impact of Severity of the disease on cost of illness and quality of life of patients with chronic obstructive pulmonary disease" J Young Pharmacists 2015;7:106.

How to cite this article: Goyal A. Effectiveness, safety and cost analysis of add-on pidotimod in maintenance therapy for exacerbation of chronic obstructive pulmonary disease in adults. Indian $\mathrm{J}$ Immunol Respir Med. 2018;3(4):203-209. 